\title{
MINIMISING CONTENT STAGNATION IN CONSTRUCTION EDUCATION: AUTOMATING THE CONTENT UPDATE THROUGH A SHARED WEB REPOSITORY FOR DISTANCE CONSTRUCTION EDUCATION
}

\author{
Kaushal Keraminiyage, \\ Dr. Dilanthi Amaratunga, \\ Dr. Richard Haigh, \\ School of the Built Environment, \\ The University of Salford, Salford, UK
}

\author{
Prof. Arturas Kaklauskas \\ Department of Construction Economics \\ and Property Management, \\ Vilnius Gediminas Technical University, \\ Vilnius, Lithuania
}

\begin{abstract}
With the use of modern information and communication technologies the distance education in construction has become easier and effective than ever before. However, the focus of the most of the construction distance education systems in place today is the need of students who are geographically prevented from attending the programmes hosted by an educational institution established physically or virtually. Generally, this arrangement is based on a model, where the information is hosted centrally by a single organisation while the geographically apart students access information asynchronously. One of the shortcomings of this model is the possibility of content stagnation due to the inflexibility in updating the content. Addressing this issue, as a part of an international collaborative research project, namely European and Asian Infrastructure Advantage (EURASIA), a virtual environment has been created where the partners (five higher education institutions from Europe and Asia) are contributing to a central content repository.
\end{abstract}

Accordingly, this paper discusses the rationale and the technology used for this virtual environment and its development process. Further it discusses its progress to date and the benefits envisaged by the users to date.

\section{KEYWORDS}

construction distance education, web technologies, shared repositories, content sharing

\section{BACKGROUND}

As the modern knowledge based economy requires its stakeholders to capture new knowledge in a timely and effective manner, innovative ways of disseminating knowledge have been experimented in the recent past, with the help of modern technological advances [4]. While distance learning is not a complete discovery of the knowledge economy, with the advent of the world wide web (www) the distance learning was repositioned as a major tool for course delivery so that knowledge can 
be disseminated within a setting of the learner's choice aligned with a pace that they can easily cope with. The advancement of the web related technologies facilitated the modern distance learning to overcome the traditional distance learning barriers such as one way communication and minimal teacher learner interaction [3]. This advancement has largely made possible through the introduction of the Virtual Learning Environments for distance learning initiatives. Virtual Learning Environments (VLEs) are predominantly used within modern Distance Learning programmes and are based on Computer Mediated Communication (CMC) tools to achieve its desired functionalities.

\section{DISTANCE LEARNING THEORIES}

Garrison [1] conducted a detail literature review of the historical perspective of distance learning. According to this review, in early 70's the focus of the distance learning was shifted from 'correspondent study' focus towards an 'independent study' focus. This shift reflects two contrasting schools of thoughts in instructional models, the symbol processing view and the situated cognition view [2]. Within the symbol processing view, the key concept is that the teacher can transmit a fixed body of information to students via an external representation. This closely related to the correspondent study view. Within this, the tutor represents an abstract idea as a concrete image and then presents the image to the learner via a medium. The learner, in turn, perceives, decodes, and stores it (Sherry, 1996). This is a tutor centred static approach, where the assumption is that the learner will be able to acquire desired knowledge externally, by processing the information or the symbols provided by the tutor. Within this setting, the required tutor - student interaction is minimal, and may have provided the basis for the radio and television based distance learning programmes. Later, this view has been further developed by adding two other dimensions to the equation, the learner's setting (environment, current situation, other sensory input) and mind (memories, associations, emotions, inference and reasoning, curiosity and interest) [2]. This enhancement argues that the knowledge is not externally transferred, but internally created using the tutor inputs and the previous knowledge gathered about the subject through various means.

The situated cognition approach is based on constructivist principles, in which a learner actively constructs an internal representation of knowledge by interacting with the material to be learned. The same is the basis for problem based learning approach [2]. This approach closely maps with the independent study focus, where the learner is the centre of the learning arrangement and the tutor student interaction is the key for the desired knowledge creation.

Despite this, in modern VLEs, the tutor -learner interaction is visible as a week link. In today's context, within a typical asynchronous DL setting, the information are hosted centrally by a single organisation allowing access for multiple users from various geographical areas. Within this arrangement the information flow is one-way. The tutor creates the Distance Learning (DL) course content within the DL environment (VLE) at the beginning of the programme delivery. The same information is then accessed by the learners asynchronously. Figure 1 below represents this arrangement.

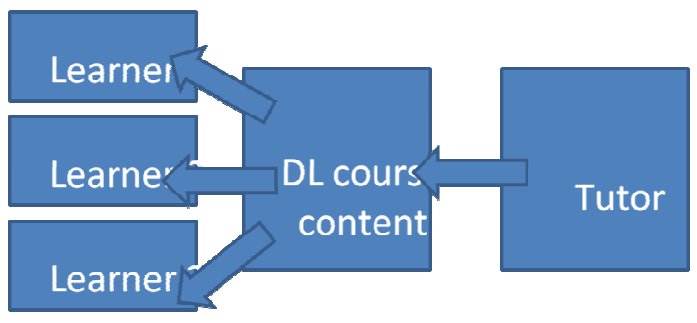

Figure 1. Learning Process

One of the shortcomings of this model is possible information and knowledge stagnation. Often, the content created by the tutor for a DL programme is repeatedly used by the tutor over and over again without an effort to update the content. This is partially due to the lack of opportunity and requirement to update tutor's knowledge on the subject as the content has a lesser chance of being challenged by the learners (due to lack of interaction between the tutor and the learners). Addressing this issue, as a part of an international collaborative research project, namely European and Asian Infrastructure Advantage (EURASIA), a virtual 
environment has been created where various parties can contribute to knowledge creation, updating, dissemination and sharing. The main advantage of this virtual environment is that, it acts as a multi functional tool within the scope of the project operating within the areas under mentioned.

Acting as a knowledge hub between partner institutions facilitating the knowledge creation, updating, sharing and dissemination

Acting as the administrative hub of the project providing the required infrastructure for the project administration

Acting as the main communication hub between the partner institutions

This virtual environment creates a "networked" model creating opportunities to update contents by various partners (five higher education institutions from Europe and Asia) who have access to the same content. In a DL environment scenario, deviating from the traditional DL model (figure 1 above) this virtual environment creates two dimensional information flows, where the centrally hosted content is continuously challenged by allowing networked access to the content by various subject experts (tutors). This model can be represented as in Figure 2 below.

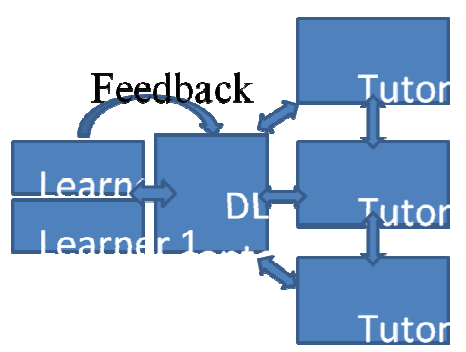

Figure 2. "Networked" model

This model presents the following advantages above the traditional DL model discussed above.

Two way information flow at the tutor's end leading to knowledge update at the tutor's end

Possible dynamic content update

Strengthened communication between tutors

Possible partnerships with peers at the tutor's end
At the same time, following issues can be highlighted related to this model.

\section{Context sensitivity}

Skills and requirements mismatches

Common issues related to joint curriculum development

With the intention of identifying and justifying the rationale behind the development of the above mentioned virtual environment, it is essential to focus on the details of the EURASIA project.

\section{THE EURASIA PROJECT}

European Commission funded research project, the EURopean and ASian Infrastructure Advantage (EURASIA) is an international collaborative research project. Five multi national project partners are working in collaboration within this project; three European higher education institutes and two Sri Lankan higher education institutes; namely the University of Moratuwa and the University of Ruhuna. The two Sri Lankan partners are specifically the leading higher education institutions that produce construction specialists for the country. The three European partners are located within United Kingdom, Estonia and Lithuania namely, the University of Salford - United Kingdom, The Tallinn University of Technology - Estonia and Vilnius Gediminas Technical University Lithuania. There have been a number of FM developments since the early 1990s, with the University of Salford being the leading UK based institution. However as highlighted above, the problem at present is that current UK provision lacks contextual knowledge on facilities and infrastructure management (particularly in association with the natural disasters such as Tsunami) in the South Asian developing economy. Collaboration with Sri Lankan institutions will increase the relevance of such programmes with up-do-date embedded case studies. Both Sri Lankan partners are located within the tsunami affected areas; one being situated within the worst affected southern province will be able to contribute to the development of case study material in support of the programme. This will be a direct contribution towards support for implementation of capacity building. 
In addition to the benefit to the Asian partners, this collaboration is beneficial from the European partners creating a vital win - win situation. That in effect creates the important mutual benefit basis which forms an important part of any collaborative work.

\section{EURASIA AIMS AND OBJECTIVES}

The main aim of the EUARSIA project is to foster cooperation in Higher Education institutions in both Europe and Asia, improve reciprocal understanding of cultures, exchange best practice and strengthen mutual awareness of programs specifically related to disaster recovery management and capacity building. The specific objective of the project is to enhance the capacity of the partner institutions for training, teaching and research activities required for the creation and long-term management of public and commercial facilities and elements of infrastructure associated with post-Tsunami activities in Sri Lanka. The project will achieve this by: developing and improving the Sri Lankan and EU's staff and postgraduate students' professional and research skills associated with the creation and management of facilities and infrastructure utilising the teaching experience of the EU University partners to develop a curriculum on the creation and long term management of public and commercial facilities and elements of infrastructure; improving and consolidating academic networks by encouraging systematic exchanges so as to establish a sustainable link between EU and Sri Lankan partner Universities; developing joint institutional systems and procedures for the provision and monitoring of training, teaching and research activities associated with the creation and management of facilities and infrastructure; providing career development opportunities to junior staff through postgraduate study and training programmes with partner Universities; and, disseminating knowledge and interpreting information through joint publications and by conducting lectures, seminars, workshops and conferences.

To achieve these aims and objectives, the necessity of creating a collaborative research environment has been identified. Further the multi national, geographically dispersed partner institutions has demanded this research environment to carry capabilities beyond traditional collaborative working environments, resulting investigations to identify how to create collaborative research environments beyond geographical and cultural limitations. These investigations resulted in the creation of the Virtual Environment of the Built Environment Research (VEBER).

With reference to the background above aim of this paper can be established as to present the process and the methodology followed when creating VEBER, along with establishing its current success through the presentation of its first user evaluation results.

The next section discusses the VEBER concept and related technologies.

\section{THE VIRTUAL ENVIRONMENT FOR BUILT ENVIRONMENT RESEARCH}

VEBER based on an open source distributed toolset, known as Moodle (www.moodle.org). Moodle is an open source web based toolset developed as a Virtual Learning Environment (VLE). VEBER is developed taking this toolset as the basis and by adding and altering functionalities required for a virtual research environment.

Moodle is technically a dynamic data driven web application written in PHP and supports number of different Database Management Systems (DBMSs) as the backend. For the purpose of VEBER, MySQL is used as the backend.

Moodle (and hence, VEBER) does not demand special skills for its usage; a general knowledge on how to handle an internet browser will be sufficient for this purpose. VEBER provides the project with a broad range of functionality that facilitates administration and coordination of the EURASIA project:

Collaboration and Communication support: Traditional communication modes are less capable of meeting the demands of multiple partner institutions and therefore a set of communication tools embedded in VEBER is used for this purpose, including communication channels such as announcements, discussions, emails, forums and chat;

Information Handling and Exchange support: A file hosting tool provides a robust platform for 
information and document handling and exchange. VEBER is equipped with file hosting and sharing mechanisms including a common document repository and a private workspace to keep and share private and confidential documents securely.

Project Management support: Administration across partners are supported with calendar, tasks and notification tools of the VEBER

Data Collection and Dissemination support: To overcome potential geographical barriers, the VEBER provides a survey tool to aid data collection during the project and a file hosting tool to facilitate joint paper preparations

Research Training support: VEBER provides discussion forums, file hosting, chat, online workshops and related learning materials, and library services, for sharing materials.

\section{THE DEVELOPMENT PROCESS}

The development process of VEBER was started by identifying the resource requirements in terms of hardware and Software. The main requirement was to purchase a suitable web server along with proper network infrastructure. A detailed hardware requirement assessment was conducted to identify the suitable hardware profile of the server to be purchased.

In addition following items and services have been used during the development process.

1 Gigabit Ethernet port with a fixed Internet Protocol (IP) address to provide the server with a fast connection to the internet

A domain registration (http://veber.buhu.salford.ac. uk) and appropriate Domain Name Service (DNS) entries to access the server over the internet. A dedicated email address for the project was also created at this point (eurasia@salford.ac.uk)

IP level firewall protection to minimize possible malicious attacks to the server

The second stage of the development process was to install appropriate software for the server and configure it to ensure optimum performance levels and maximum security. Microsoft Windows Server 2003 has been selected as the operating systems for the server and inbuilt Internet Information Service (IIS) has been used as the web server software to host VEBER as a web application.

The next step was to acquire the Moodle toolset to be installed in the server as the basis for the VEBER. This toolset is available to be downloaded free of charge from http://www.moodle.org and has been released under the General Public License (GPL) which allowed us to use and modify the programme to suite the requirements of the project. As PHP and MySQL are prerequisites for the installation of Moodle, those were obtained (under GPL) and installed in the server prior installing the Moodle.

After installing and testing Moodle, the toolset has been modified to create the desired environment and functionalities of the proposed VRE. Specifically, three major changes were introduced to the Moodle toolset during the phase 2 of the VEBER implementation

Created a new language pack (named as "en-re") to reflect the changes to the vocabulary / terminology used within VEBER: e.g. the term "teacher" is Moodle needed to be changed to "research project facilitator" in VEBER.

Created a new "course format" (named as "my format") to implement the structural changes made in VEBER: e.g. The "meta courses" and "courses" concepts in Moodle have been redefined in VEBER to reflect the "research projects" and "work packages"

Created a new theme (named as "mystyle") to create the desired visual impact.

In addition to these major changes, the VEBER has been updated continuously by modifying the base Moodle code and adding functions to it. These include:

Developing some custom 'blocks' for various desired functionalities (e.g. My Research Projects and File sharing)

Developing and testing an integration methodology to use Moodle integrated virus scans (calmav based to protect file uploads) and Moodle integrated spell checkers (aspell) within a Windows 2003 environment. Even though these tools are available 
under the standard Moodle installation, these tools are primarily designed for a *nux environment. Within the development of VEBER these tools were attempted to integrate within a Windows environment and the new knowledge and experience related to that attempt has been shared with the Moodle community.(see: http://moodle.org/mod/ forum/discuss.php?d=25843)

\section{CONCLUSION}

Traditional asynchronous distance learning model has its drawbacks related to possible content stagnation. One of the reasons behind this is the rigid approach used within the model to create and disseminate content by the tutor. Within this arrangement the content of created by the tutor is not subjected to peer challenges creating opportunities for possible content stagnation. The "network" DL model addresses this shortcoming through a central content repository created as a virtual research environment as a part of the EURASIA project. EURASIA is a collaborative research project between five European and Asian higher education institutions. The Virtual Research Environment Created above is named as VEBER (Virtual Research Environment for Built Environment Research). This paper discussed the rationale, the methodology and the technology behind the development of the VEBER.

\section{ACKNOWLEDGEMENT}

The authors wish to acknowledge that the EURASIA project is funded by the European Commission under the Asia - Link program. Further, this paper has been produced with the financial assistance of the European Union. The contents of this paper are the sole responsibility of EURASIA and can under no circumstance be regarded as representing the position of the European Union.

\section{REFERENCES AND BIBLIOGRAPHY}

[1] Garrison, G. (2000), "Theoretical challenges for distance education in the $21^{\text {st }}$ century: A shift from structural to transactional issues", International review of research in open and distance learning, Volume 1.1, pp $1-17$

[2] Sherry, L. (1996). "Issues in Distance Learning." International Journal of Education 1(4): 337-365.

[3] Terzi, S., Celik, A (2003) "Teacher-Student Interactions In Distance Learning", International Education Technology Conference and Fair, North Cyprus

[4] Zhang, Dongsong; Zhao, J. Leon; Zhou, Lina; and Nunamaker, Jay F. Jr. "Can E-Learning Replace Classroom Learning?" Communications of the ACM 47 , no. 5 (2004): 75-79. 